\title{
A review of studies using the Körperkoordinationstest für Kinder (KTK)
}

\author{
Susanna Iivonen, Arja Kaarina Sääkslahti, \& Arto Laukkanen \\ Department of Sport Sciences, University of Jyväskylä, Finland
}

\begin{abstract}
The Körperkoordinationstest für Kinder (KTK) (Kiphard \& Schilling, 1974, 2007) is a standardized, norm-referenced measure used by physical therapists and occupational therapists in clinic and school practice settings to evaluate the motor coordination (MC) of 5- to 14-year-old children. To find out for which research purposes the KTK has been used and its reliability and validity, we conducted a systematic review. Searching five databases, we found 46 studies used the KTK over four decades. The KTK was widely used in Europe in children with typical and atypical development. The KTK was used to investigate associations, to test the effects of interventions and treatments, to identify or diagnose different factors, to evaluate $\mathrm{MC}$ and it was included in reviews of motor assessments. The KTK was reported to be a reliable measure, although only nine studies examined its validity or reliability in target populations. The KTK was considered a non-sport/skill-specific, easy-to-administer, had a scoring system that enabled crossstudy comparisons but was limited to balance and locomotor aspects, had norm values that were outdated, and needed careful standardization. The KTK's validity for different purposes requires further evaluation.
\end{abstract}

Keywords: test battery, literature review

\section{Introduction}

In the 1960s, German child and youth psychiatrists E. J. Kiphard, and F. Schilling were interested in devising, for use in treating psychomotor deficits, a practical test that would indicate an individual's capacity for sensory-motor integration in order to control and coordinate the body (Kiphard and Schilling, 2007, 7-9). Children with brain disorders, in particular, seemed to have major difficulties in achieving the sensory-motor integration required to coordinate their bodies. If these deficits could be recognized early enough, a child could receive rehabilitation that would reduce negative compensation strategies and enable him or her to become integrated into society. After scrutinizing 150 activity test options and examinations administered to children, Kiphard and his German colleagues created the HammMarburger Körperkoordinationstests, comprising six test items. After each test item was evaluated theoretically and in practice, the final Körperkoordinationstest für Kinder (KTK) with four test items was established
(Kiphard and Schilling, 1974, 2007). The test items are described in the Supplementary file 1 (Supplementary file 1).

Studies on the quality criteria and standardization of KTK were conducted in 1973-74 among children aged 5 to 14 . The children comprised the 1228 standardization sample (the KTK norm group), 79 with confirmed brain disorder, 59 with suspected brain disorder, 79 with behavior disturbances, and 27 with speech disturbances. For the raw scores on the four test items, the test-retest reliability coefficients ranged between $0.80 \geq \mathrm{r}$ $\leq 0.96$, and for the total raw score, the reliability was 0.97 . Validity was indicated through differentiation between children with typical and atypical development or disability. With the KTK, $91 \%$ of children with brain damage could be differentiated from children with typical development. The explained variance of the total score by the four test items ranged from $81 \%$ (age 6) to $98 \%$ (age 9 ), indicating high content validity. Internal structure validity according to factor analysis indicated that the four test items loaded on the same factor. The intercorrelations between the 
four items ranged from 0.60 (WB/JS) to 0.81 (HH/JS).

The KTK has a long history and, in practice, we found that it is referred to quite a lot in the literature related to the study of childhood motor development. This allows us to hypothesize that the KTK is widely used today and adopted in disciplines and for purposes other than it was originally intended. If these hypotheses hold true, it is essential to analyze for which research purposes the KTK has been used and the reliability and validity of the KTK in these different studies. The purpose of our review was to analyze the use of the KTK in different studies by (1) describing the characteristics of the studies, (2) categorizing them according to their main purpose(s) and utilization of the KTK, and (3) describing the validity and reliability of the KTK in the target populations appended with the users' comments.

\section{Methods}

A systematic search of the literature to identify articles that reported on use of the KTK was conducted in January 2014 and updated in February 2015. We sourced the articles from the following databases: MEDLINE (ProQuest), PsycINFO (ProQuest), SPORTDiscus with Full Text (EBSCO), PubMed (Medline) and Web of Science (WoS). We used the search terms "KTK OR Körperkoordinations test OR Körperkoordinationstest" in the search strategy. We included studies if (1) a search term existed in the title or abstract, (2) the search term "KTK" did not refer to something other than the Körperkoordinations test or Körperkoordinationstest, and (3) the article had been published in an English- or Germanlanguage peer-reviewed journal.

The first author omitted duplicates from the lists of titles of the identified articles. The first two authors then independently checked the titles and the abstracts to arrive at the selection of articles to be reviewed. For reliability, we calculated the percentage of agreement between the two reviewers. One hundred percent agreement was obtained. The first author read the included articles, extracted the material pertaining to the purpose of this review, and created a detailed summary table. The authors utilized the table when they categorized the articles according to their main purposes and use of the KTK test items in populations with typical development and with special needs. To establish the validity and reliability of the KTK and conclusions for using it for research purposes, we utilized the summary table and examined the whole texts for more exact information. Finally, we screened the reference lists of the retrieved articles for possible inclusion. All disagreements were resolved by consensus. This study was approved by the University of Jyväskylä. The articles included in this review were published in public academic journals.

\section{Results}

\section{Characteristics of studies}

A total of 192 potential titles were identified through the database searches: MEDLINE (ProQuest) 34, PubMed (Medline) 77, PsycINFO (ProQuest) 18, SPORTDiscus with Full Text (EBSCO) 12, and Web of Science - WoS 51. After duplicates were removed, a total of 42 articles satisfied the inclusion criteria. After an updated literature search across the five databases, we accepted four more articles, making a total of 46 articles for this review. An overview of the studies is shown in Table 1. A detailed table of the studies is available upon request from the first author. 
Table 1. Summary of the included studies.

\begin{tabular}{|c|c|c|c|c|c|c|}
\hline $\begin{array}{l}\text { First author } \\
\text { (year), location }\end{array}$ & Language & Topic & Design & $\begin{array}{l}\text { Participants: age, } \\
\text { grouping }\end{array}$ & \begin{tabular}{|l} 
The KTK \\
scoring used
\end{tabular} & Discipline \\
\hline $\begin{array}{l}\text { Neühauser } \\
(1975) \\
\text { Germany }\end{array}$ & German & $\begin{array}{l}\text { Review of } \\
\text { psychomotor tests }\end{array}$ & Review & $\begin{array}{l}11 \text { different } \\
\text { assessments }\end{array}$ & & Pediatry \\
\hline $\begin{array}{l}\text { Schenck } \\
(1978) \\
\text { Germany }\end{array}$ & German & $\begin{array}{l}\text { Diagnostic } \\
\text { efficiency of the } \\
\text { KTK }\end{array}$ & Longitudinal & $\begin{array}{l}N=140 \text { (the } \\
\text { comparison } \\
\text { group) } \\
N=224 \text { with } \\
\text { different } \\
\text { impairments } \\
\text { 5-12 years }\end{array}$ & $\begin{array}{l}\text { HH and JS } \\
\text { MQs }\end{array}$ & Pediatry \\
\hline $\begin{array}{l}\text { Lesigang (1982) } \\
\text { Austria }\end{array}$ & German & $\begin{array}{l}\text { Identify children } \\
\text { with minimal } \\
\text { cerebral palsy, } \\
\text { and associations } \\
\text { between } \\
\text { motometric } \\
\text { neuropsychologi } \\
\text { cal } \\
\text { investigations } \\
\text { and motor } \\
\text { coordination }\end{array}$ & $\begin{array}{l}\text { Cross- } \\
\text { sectional }\end{array}$ & $\begin{array}{l}N=192 \text { (132 } \\
\text { boys) } \\
\text { Classes 1-4 from } \\
\text { the special school } \\
\text { for children with } \\
\text { speech } \\
\text { disturbances }\end{array}$ & $\begin{array}{l}\text { MQs and } \\
\text { classification } \\
\text { into } 4 \\
\text { categories }\end{array}$ & Neurology \\
\hline $\begin{array}{l}\text { Winneke (1982) } \\
\text { Germany }\end{array}$ & English & $\begin{array}{l}\text { Associations } \\
\text { between gross } \\
\text { motor } \\
\text { coordination, } \\
\text { lead-exposure } \\
\text { and intellectual } \\
\text { performance }\end{array}$ & $\begin{array}{l}\text { Cross- } \\
\text { sectional, } \\
\text { comparative } \\
\text { study, } \\
\text { pair matched }\end{array}$ & $\begin{array}{l}N=52 \\
\text { Mean age } 8.5 \\
\text { years } \\
26 \text { with tooth-lead } \\
\text { level }(\mathrm{PbT}< \\
3 \mathrm{ppm})\end{array}$ & MQs & Pediatry \\
\hline $\begin{array}{l}\text { Schneider } \\
(1984) \\
\text { United States }\end{array}$ & German & $\begin{array}{l}\text { Effectiveness of } \\
\text { intervention } \\
\text { program on } \\
\text { overall body } \\
\text { coordination in } \\
\text { dyslexic boys }\end{array}$ & $\begin{array}{l}\text { Longitudinal } \\
(7.5 \text { weeks }) \\
\text { with pre- and } \\
\text { post- } \\
\text { measurement } \\
\mathrm{s}\end{array}$ & $\begin{array}{l}N=40 \text { boys } \\
12 \text { year-olds } \\
20 \text { dyslexic }\end{array}$ & $\begin{array}{l}\text { Raw scores } \\
\text { and MQs }\end{array}$ & Education \\
\hline $\begin{array}{l}\text { Camacho-Araya } \\
(1990) \\
\text { Costa Rica }\end{array}$ & English & $\begin{array}{l}\text { Assessment of } \\
\text { stability and } \\
\text { internal } \\
\text { reliability of the } \\
\text { Spanish version } \\
\text { of the KTK }\end{array}$ & $\begin{array}{l}\text { Reliability } \\
\text { study }\end{array}$ & $\begin{array}{l}N=280 \\
\text { Kindergarteners } \\
\text { and } 1^{\text {st }} \text { and } 2^{\text {nd }} \\
\text { graders }\end{array}$ & \begin{tabular}{|l|} 
Original \\
KTK items
\end{tabular} & Sport sciences \\
\hline $\begin{array}{l}\text { Smits- } \\
\text { Engelsman } \\
(1998) \\
\text { The Netherlands }\end{array}$ & English & $\begin{array}{l}\text { Suitability and } \\
\text { comparison of } \\
\text { the norms of } \\
\text { Movement ABC } \\
\text { and the KTK } \\
\end{array}$ & $\begin{array}{l}\text { Cross- } \\
\text { sectional }\end{array}$ & $\begin{array}{l}N=208 \text { ( } 87 \text { boys }) \\
5-13 \text { years } \\
74 \text { with suspected } \\
\text { motor problems } \\
\text { (5-12 years) }\end{array}$ & $\begin{array}{l}\text { KTK total } \\
\text { scores }\end{array}$ & Pediatry \\
\hline $\begin{array}{l}\text { Jöhnk } \\
\text { (1999) } \\
\text { Germany }\end{array}$ & English & $\begin{array}{l}\text { Introduction of } \\
\text { methods for the } \\
\text { evaluation of } \\
\text { sensorimotor } \\
\text { functions after } \\
\text { traumatic brain } \\
\text { injury }\end{array}$ & $\begin{array}{l}\text { Discussion of } \\
9 \text { methods, } \\
\text { longitudinal } \\
\text { follow-up } \\
\text { case-study }\end{array}$ & $\begin{array}{l}N=17 \\
\text { One } 11 \text {-years-old } \\
\text { girl suffering } \\
\text { from severe } \\
\text { traumatic brain } \\
\text { injury }\end{array}$ & MQ & Neuropediatry \\
\hline $\begin{array}{l}\text { Stieh } \\
(1999) \\
\text { Germany }\end{array}$ & English & $\begin{array}{l}\text { Determine gross } \\
\text { motor } \\
\text { coordination of } \\
\text { children with } \\
\text { heart disease }\end{array}$ & $\begin{array}{l}\text { Cross- } \\
\text { sectional }\end{array}$ & $\begin{array}{l}N=132 \text { (67 boys) } \\
5-14 \text { years } \\
102 \text { (53 boys) with } \\
\text { various forms of } \\
\text { congenital heart } \\
\text { disease }\end{array}$ & MQs & Pediatry \\
\hline
\end{tabular}


The Körperkoordinationstest für Kinder (KTK)

\begin{tabular}{|c|c|c|c|c|c|c|}
\hline $\begin{array}{l}\text { Laucht } \\
(2000) \\
\text { Germany }\end{array}$ & German & $\begin{array}{l}\text { Developmental } \\
\text { sequel of } \\
\text { impacts of early } \\
\text { organic risk } \\
\text { (pre- and } \\
\text { perinatal } \\
\text { complications) } \\
\text { and } \\
\text { psychological } \\
\text { risk on motor, } \\
\text { cognitive and } \\
\text { socioemotional } \\
\text { outcomes }\end{array}$ & \begin{tabular}{|l} 
Cross - \\
sectional
\end{tabular} & $\begin{array}{l}N=347(171 \\
\text { boys }) \\
\text { prospective cohort }\end{array}$ & JS & Pediatry \\
\hline $\begin{array}{l}\text { May } \\
(2001) \\
\text { Germany }\end{array}$ & German & $\begin{array}{l}\text { Effects of regular } \\
\text { Judo training }\end{array}$ & $\begin{array}{l}\text { Intervention, } \\
\text { Prospective } \\
\text { controlled } \\
\text { design }\end{array}$ & $\begin{array}{l}N=33 \text { boys } \\
13-26 \text { years } \\
14 \text { in judo group } \\
\text { with multiple } \\
\text { handicaps }\end{array}$ & $\begin{array}{l}\text { Raw scores, } \\
\text { MQs }\end{array}$ & Pediatry \\
\hline $\begin{array}{l}\text { Graf } \\
(2003) \\
\text { Germany }\end{array}$ & German & $\begin{array}{l}\text { Associations of } \\
\text { overall body } \\
\text { coordination and } \\
\text { endurance } \\
\text { performance }\end{array}$ & $\begin{array}{l}\text { Cross- } \\
\text { sectional, } \\
\text { intervention }\end{array}$ & $\begin{array}{l}=906 \text { ( } 479 \\
\text { boys) } \\
6-7 \text { years } \\
668 \text { ( } 341 \text { boys) in } \\
\text { intervention } \\
\text { group }\end{array}$ & MQs & Sport sciences \\
\hline $\begin{array}{l}\text { Hebestreit } \\
(2003) \\
\text { Germany }\end{array}$ & English & $\begin{array}{l}\text { Association of } \\
\text { head size with } \\
\text { whole-body } \\
\text { coordination and } \\
\text { the oxygen cost } \\
\text { in cycling test }\end{array}$ & $\begin{array}{l}\text { Cross- } \\
\text { sectional }\end{array}$ & $\begin{array}{l}N=54 \text { (19 boys) } \\
6-12 \text { years } \\
33 \text { prematurely } \\
\text { born }\end{array}$ & MQ & Neuropediatry \\
\hline $\begin{array}{l}\text { Baumann } \\
(2004) \\
\text { Germany }\end{array}$ & German & $\begin{array}{l}\text { Asssociations of } \\
\text { ascertained } \\
\text { results and } \\
\text { motor } \\
\text { development } \\
\text { with ICD } 10\end{array}$ & $\begin{array}{l}\text { Cross- } \\
\text { sectional, } \\
\text { clinical }\end{array}$ & $\begin{array}{l}N=102 \text { (79 boys) } \\
5-13 \text { years } \\
\text { psychiatric } \\
\text { patients }\end{array}$ & MQs & Neuropediatry \\
\hline $\begin{array}{l}\text { Graf } \\
(2004) \\
\text { Germany }\end{array}$ & English & $\begin{array}{l}\text { Associations } \\
\text { between body } \\
\text { mass index } \\
\text { (BMI), PA and } \\
\text { different motor } \\
\text { tests of gross } \\
\text { motor } \\
\text { development }\end{array}$ & $\begin{array}{l}\text { Cross- } \\
\text { sectional }\end{array}$ & $\begin{array}{l}N=556(281 \\
\text { boys }) \\
\text { Mean age } 6.7 \\
\text { years }\end{array}$ & MQs & Sport sciences \\
\hline $\begin{array}{l}\text { Prätorius (2004) } \\
\text { Germany }\end{array}$ & German & $\begin{array}{l}\text { Differences in } \\
\text { coordinative } \\
\text { performances } \\
\text { between children } \\
\text { from } \\
\text { socioeconomical } \\
\text { ly diverse } \\
\text { settings }\end{array}$ & $\begin{array}{l}\text { Cross- } \\
\text { sectional }\end{array}$ & $\begin{array}{l}N=163(85 \text { boys }) \\
6-13 \text { years }\end{array}$ & MQs & Sport sciences \\
\hline $\begin{array}{l}\text { Steiss } \\
(2005) \\
\text { Germany }\end{array}$ & German & $\begin{array}{l}\text { Comparison of } \\
\text { neurodevelopme } \\
\text { nt profiles } \\
\text { examined by } \\
\text { different tests } \\
\end{array}$ & $\begin{array}{l}\text { Cross- } \\
\text { sectional }\end{array}$ & $\begin{array}{l}N=58 \text { (26 boys) } \\
9-12 \text { years } \\
31 \text { preterm infants }\end{array}$ & MQs & Neuropediatry \\
\hline $\begin{array}{l}\text { Cools } \\
(2009) \\
\text { Belgium }\end{array}$ & English & $\begin{array}{l}\text { Comparison of } \\
\text { seven movement } \\
\text { skill assessment } \\
\text { tools }\end{array}$ & Review & $\begin{array}{l}\text { The reviewed } \\
\text { tools: } \\
\text { MOT 4-6 } \\
\text { Movement-ABC } \\
\text { PDMS-2 } \\
\text { KTK } \\
\text { TGMD-2 } \\
\text { MMT } \\
\text { BOTMP-BOT-2 }\end{array}$ & - & Sport Sciences \\
\hline
\end{tabular}




\begin{tabular}{|c|c|c|c|c|c|c|}
\hline $\begin{array}{l}\text { Gheysen } \\
(2008) \\
\text { Belgium }\end{array}$ & English & $\begin{array}{l}\text { Impact of } \\
\text { implant on the } \\
\text { motor } \\
\text { development of } \\
\text { deaf children } \\
\end{array}$ & Experimental & $\begin{array}{l}N=79 \text { (30 boys) } \\
4-12 \text { years } \\
36 \text { deaf }\end{array}$ & MQs & Pediatry \\
\hline $\begin{array}{l}\text { Hanewinkel-van } \\
\text { Kleef (2009) } \\
\text { The Netherlands }\end{array}$ & English & $\begin{array}{l}\text { Predictors of } \\
\text { motor } \\
\text { performance in } \\
\text { children with } \\
\text { joint } \\
\text { hypermobility } \\
\text { and motor } \\
\text { problems } \\
\end{array}$ & $\begin{array}{l}\text { Cross- } \\
\text { sectional, } \\
\text { descriptive }\end{array}$ & $\begin{array}{l}N=41 \text { ( } 22 \text { boys }) \\
5-12 \text { years }\end{array}$ & Percentiles & Pediatry \\
\hline $\begin{array}{l}\text { Van Aken } \\
(2009) \\
\text { The Netherlands }\end{array}$ & English & $\begin{array}{l}\text { Determinate } \\
\text { motor } \\
\text { performance of } \\
\text { 22q11.(chromos } \\
\text { ome) deletion } \\
\text { syndrome } \\
\text { children }\end{array}$ & $\begin{array}{l}\text { Cross- } \\
\text { sectional, } \\
\text { descriptive }\end{array}$ & $\begin{array}{l}N=56 \text { (28 boys) } \\
\text { Mean age } 9.6 \\
\text { years } \\
28 \text { in } 22 q 11 . D S \\
\text { group }\end{array}$ & MQs & Pediatry \\
\hline $\begin{array}{l}\text { Martins } \\
\text { (2010) Portugal, } \\
\text { Azores Islands }\end{array}$ & English & $\begin{array}{l}\text { Longitudinal } \\
\text { associations and } \\
\text { changes in gross } \\
\text { motor } \\
\text { coordination, } \\
\text { BMI, PA and } \\
\text { fitness during } 5 \\
\text { consecutive } \\
\text { years }\end{array}$ & $\begin{array}{l}\text { Longitudinal } \\
\text { (5 years) }\end{array}$ & $\begin{array}{l}N=285(143 \\
\text { boys }) \\
6-10 \text { years }\end{array}$ & $\begin{array}{l}\text { Sum of the } 4 \\
\text { tests' scores } \\
\text { adjusted for } \\
\text { age and sex }\end{array}$ & Sport sciences \\
\hline $\begin{array}{l}\text { Toftegaard- } \\
\text { Stoeckel } \\
\text { (2010) } \\
\text { Denmark }\end{array}$ & English & $\begin{array}{l}\text { Associations } \\
\text { between } \\
\text { children's } \\
\text { physical } \\
\text { competence, } \\
\text { bodily } \\
\text { competence, } \\
\text { BMI, and motor } \\
\text { abilities, and } \\
\text { comparison } \\
\text { with the KTK } \\
\text { normative } \\
\text { material } \\
\end{array}$ & \begin{tabular}{|l|} 
Cross- \\
sectional
\end{tabular} & $\begin{array}{l}N=646(338 \\
\text { boys }) \\
6-7 \text { years }\end{array}$ & MQ & Sport sciences \\
\hline $\begin{array}{l}\text { D'Hondt } \\
\text { (2011b) } \\
\text { Belgium }\end{array}$ & English & $\begin{array}{l}\text { Short-term (2 } \\
\text { years) } \\
\text { effectiveness of } \\
6-10 \text { months } \\
\text { obesity/overweig } \\
\text { ht treatment } \\
\text { program }\end{array}$ & $\begin{array}{l}\text { Intervention } \\
\text { Quasi } \\
\text { Experimental, } \\
\text { matched pairs }\end{array}$ & $\begin{array}{l}N=72 \text { ( } 48 \text { boys }) \\
7-13 \text { years } \\
36 \\
\text { overweight/obese }\end{array}$ & $\begin{array}{l}\text { Raw scores } \\
\text { and MQ }\end{array}$ & Pediatry \\
\hline $\begin{array}{l}\text { D'Hondt } \\
\text { (2011a) } \\
\text { Belgium }\end{array}$ & English & $\begin{array}{l}\text { Differences in } \\
\text { gross motor } \\
\text { coordination in } \\
\text { healthy-weight, } \\
\text { overweight and } \\
\text { obese children }\end{array}$ & \begin{tabular}{|l|} 
Cross- \\
sectional
\end{tabular} & $\begin{array}{l}N=954(454 \\
\text { boys }) \\
5-12 \text { years }\end{array}$ & MQ & Pediatry \\
\hline $\begin{array}{l}\text { Graf } \\
(2011) \\
\text { Germany }\end{array}$ & German & $\begin{array}{l}\text { Effects of the } \\
\text { four-year-long } \\
\text { Children's } \\
\text { Health } \\
\text { Interventional } \\
\text { Trial (CHILT) - } \\
\text { project on motor } \\
\text { productivity and } \\
\text { whole body } \\
\text { coordination }\end{array}$ & $\begin{array}{l}\text { Cluster- } \\
\text { randomized- } \\
\text { controlled- } \\
\text { intervention- } \\
\text { trial }\end{array}$ & $\begin{array}{l}N=615 \\
6-7 \text { years } \\
436 \text { in } \\
\text { intervention } \\
\text { schools }\end{array}$ & MQs & Sport sciences \\
\hline
\end{tabular}


The Körperkoordinationstest für Kinder (KTK)

\begin{tabular}{|c|c|c|c|c|c|c|}
\hline $\begin{array}{l}\text { Kranz } \\
(2011) \\
\text { Switzerland }\end{array}$ & German & $\begin{array}{l}\text { Effects of a } \\
\text { grapho- and } \\
\text { psychomotor } \\
\text { treatment on } \\
\text { motor } \\
\text { coordination } \\
\text { index } \\
\end{array}$ & $\begin{array}{l}\text { Experimental } \\
\text { longitudinal } \\
\text { with pre-, } \\
\text { post-, and } \\
\text { follow-up- } \\
\text { test }\end{array}$ & $\begin{array}{l}N=188 \\
\text { Mean age 4.1years } \\
68 \text { in experimental } \\
\text { group }\end{array}$ & Index-KTK & Sport sciences \\
\hline $\begin{array}{l}\text { Krombholz } \\
(2011) \\
\text { Germany }\end{array}$ & German & $\begin{array}{l}\text { Changes in motor } \\
\text { performance } \\
\text { between years } \\
2001 \text { and } \\
1973 / 1974 \text { and } \\
1977\end{array}$ & $\begin{array}{l}\text { Retrospective, } \\
\text { cohorts }\end{array}$ & $\begin{array}{l}N=375 \text { in "Studie } \\
1973 ", 3-6 \text { years } \\
N=123 \text { in "Studie } \\
1974 ", 5-6 \text { years } \\
N=644 \text { in "Studie } \\
1977 ", 6-7 \text { years } \\
N=1532 \text { in } \\
\text { "Studie } 2000 ", 3- \\
7 \text { years }\end{array}$ & $\begin{array}{l}\text { WB and JS } \\
\text { raw scores }\end{array}$ & Sport sciences \\
\hline $\begin{array}{l}\text { V. P. Lopes } \\
\text { (2011) } \\
\text { Portugal, } \\
\text { Azores Islands }\end{array}$ & English & $\begin{array}{l}\text { Longitudinal } \\
\text { relationships } \\
\text { among motor } \\
\text { proficiency, } \\
\text { physical fitness } \\
\text { and PA in 6- to } \\
10 \text {-year-old } \\
\text { children }\end{array}$ & $\begin{array}{l}\text { Longitudinal } \\
\text { (5 years) }\end{array}$ & $\begin{array}{l}N=285(143 \\
\text { boys }) \\
6-10 \text { years }\end{array}$ & MQ & Sport sciences \\
\hline $\begin{array}{l}\text { Vandorpe } \\
(2011) \\
\text { Belgium }\end{array}$ & English & $\begin{array}{l}\text { Gender- and age- } \\
\text { specific } \\
\text { reference values } \\
\text { for the gross } \\
\text { motor } \\
\text { coordination of } \\
\text { Flemish } \\
\text { children, } \\
\text { compared with } \\
\text { the norms of the } \\
\text { original German } \\
\text { standardization } \\
\text { sample }\end{array}$ & $\begin{array}{l}\text { Cross- } \\
\text { sectional }\end{array}$ & $\begin{array}{l}N=2470(1297 \\
\text { boys }) \\
6-12 \text { years }\end{array}$ & $\begin{array}{l}\text { Raw scores, } \\
\text { MQ }\end{array}$ & Sport sciences \\
\hline $\begin{array}{l}\text { Vandendriessch } \\
\text { e (2011) } \\
\text { Belgium }\end{array}$ & English & $\begin{array}{l}\text { Variance in } \\
\text { motor } \\
\text { coordination by } \\
\text { maturity, body } \\
\text { size and fitness }\end{array}$ & $\begin{array}{l}\text { Cross- } \\
\text { sectional }\end{array}$ & $\begin{array}{l}N=613 \text { boys } \\
181 \text { aged } 7 \text { years } \\
245 \text { aged } 9 \text { years } \\
187 \text { aged } 11 \text { years }\end{array}$ & Raw scores & Sport sciences \\
\hline $\begin{array}{l}\text { Debraband } \\
(2012) \\
\text { Belgium }\end{array}$ & English & $\begin{array}{l}\text { Age-related } \\
\text { effects and } \\
\text { correlations in } \\
\text { timing abilities } \\
\text { and conditions } \\
\text { with motor skill } \\
\text { proficiency }\end{array}$ & $\begin{array}{l}\text { Cross- } \\
\text { sectional }\end{array}$ & $N=80$ (40 boys) & JS & Sport sciences \\
\hline $\begin{array}{l}\text { L. Lopes } \\
(2012) \\
\text { Portugal }\end{array}$ & English & $\begin{array}{l}\text { Relationship } \\
\text { between } \\
\text { sedentary } \\
\text { behaviour and } \\
\text { motor } \\
\text { coordination }\end{array}$ & $\begin{array}{l}\text { Cross- } \\
\text { sectional }\end{array}$ & $\begin{array}{l}N=123(103 \\
\text { boys }) \\
9-10 \text { years }\end{array}$ & MQ & Sport sciences \\
\hline $\begin{array}{l}\text { V. P. Lopes } \\
\text { (2012a) } \\
\text { Portugal, } \\
\text { Azores Islands }\end{array}$ & English & $\begin{array}{l}\text { Motor } \\
\text { coordination, } \\
\text { physical fitness } \\
\text { and PA as } \\
\text { predictors of } \\
\text { the development } \\
\text { of subcutaneous } \\
\text { adiposity }\end{array}$ & $\begin{array}{l}\text { Longitudinal } \\
\text { (5 years } \\
\text { 2002-2007) }\end{array}$ & $\begin{array}{l}N=285(143 \\
\text { boys }) \\
6-10 \text { years }\end{array}$ & MQ & Sport sciences \\
\hline $\begin{array}{l}\text { V. P. Lopes } \\
\text { (2012b) } \\
\text { Portugal }\end{array}$ & English & $\begin{array}{l}\text { Association } \\
\text { between motor } \\
\text { coordination and }\end{array}$ & $\begin{array}{l}\text { Cross- } \\
\text { sectional }\end{array}$ & $\begin{array}{l}N=7175(3616 \\
\text { boys) } \\
6-14 \text { years }\end{array}$ & MQs & Sport sciences \\
\hline
\end{tabular}




\begin{tabular}{|c|c|c|c|c|c|c|}
\hline & & $\begin{array}{l}\text { BMI across } \\
\text { childhood and } \\
\text { early } \\
\text { adolescence } \\
\end{array}$ & & & & \\
\hline $\begin{array}{l}\text { Vandendriessch } \\
\text { e (2012) } \\
\text { Belgium }\end{array}$ & English & $\begin{array}{l}\text { Associations of } \\
\text { young soccer } \\
\text { players maturity } \\
\text { status with their } \\
\text { morphological } \\
\text { measures, } \\
\text { fitness, soccer- } \\
\text { specific and non- } \\
\text { specific motor } \\
\text { coordination } \\
\text { skills }\end{array}$ & $\begin{array}{l}\text { Experimental, } \\
\text { descriptive }\end{array}$ & $\begin{array}{l}N=78 \text { youth } \\
\text { national soccer } \\
\text { team players } \\
15-16 \text { years }\end{array}$ & WB, JS, MS & Sport sciences \\
\hline $\begin{array}{l}\text { D'Hondt (2013) } \\
\text { Belgium }\end{array}$ & English & $\begin{array}{l}\text { Short-term } \\
\text { evolution in } \\
\text { gross motor } \\
\text { coordination } \\
\text { according to } \\
\text { weight status, } \\
\text { and identifying } \\
\text { factors, and } \\
\text { prediction of } \\
\text { future gross } \\
\text { motor } \\
\text { coordination }\end{array}$ & $\begin{array}{l}\text { Longitudinal } \\
\text { (2 years } \\
\text { 2007-2009), } \\
\text { matched pairs }\end{array}$ & $\begin{array}{l}N=100 \text { ( } 48 \text { boys }) \\
6-10 \text { years } \\
50 \text { overweight } \\
\text { /obese }\end{array}$ & MQs & Sport sciences \\
\hline $\begin{array}{l}\text { Laukkanen } \\
\text { (2013) } \\
\text { Finland }\end{array}$ & English & $\begin{array}{l}\text { Relationship } \\
\text { between gross } \\
\text { motor skills and } \\
\text { PA analyzed } \\
\text { with two } \\
\text { different } \\
\text { methods }\end{array}$ & $\begin{array}{l}\text { Cross- } \\
\text { sectional }\end{array}$ & $\begin{array}{l}N=84(38 \text { boys }) \\
5-8 \text { years }\end{array}$ & $\mathrm{MC}$ & Sport sciences \\
\hline $\begin{array}{l}\text { V. P. Lopes } \\
\text { (2013) } \\
\text { Portugal }\end{array}$ & English & $\begin{array}{l}\text { Associations } \\
\text { between motor } \\
\text { coordination and } \\
\text { weight status, } \\
\text { and relative risk } \\
\text { of being } \\
\text { overweight/obes } \\
\text { e based on the } \\
\text { level of motor } \\
\text { coordination }\end{array}$ & $\begin{array}{l}\text { Cross- } \\
\text { sectional }\end{array}$ & $\begin{array}{l}N=6625(3344 \\
\text { boys }) \\
6-11 \text { years }\end{array}$ & MQs & Sport sciences \\
\hline $\begin{array}{l}\text { L. Lopes } \\
\text { (2013) } \\
\text { Portugal }\end{array}$ & English & $\begin{array}{l}\text { Relationship } \\
\text { between gross } \\
\text { motor } \\
\text { coordination and } \\
\text { academic } \\
\text { achievement } \\
\text { after adjusting } \\
\text { for } \\
\text { cardiorespiratory } \\
\text { fitness, BMI and } \\
\text { sociodemographi } \\
\text { cs }\end{array}$ & $\begin{array}{l}\text { Cross- } \\
\text { sectional }\end{array}$ & $\begin{array}{l}\mathrm{N}=596(315 \\
\text { boys }) \\
9-12 \text { years }\end{array}$ & MQs & Sport sciences \\
\hline $\begin{array}{l}\text { Martin-Diener } \\
\text { (2013) } \\
\text { Switzerland }\end{array}$ & English & $\begin{array}{l}\text { Correlations } \\
\text { between } \\
\text { retrospectively } \\
\text { assessed injury } \\
\text { risk incidence } \\
\text { rate, PA, aerobic } \\
\text { fitness and } \\
\text { motor } \\
\text { coordination }\end{array}$ & $\begin{array}{l}\text { Cross- } \\
\text { sectional }\end{array}$ & $\begin{array}{l}N=249(126 \\
\text { boys }) \\
\text { Mean age } 7.9 \\
\text { years }\end{array}$ & MQs & Sport sciences \\
\hline
\end{tabular}




\begin{tabular}{|c|c|c|c|c|c|c|}
\hline $\begin{array}{l}\text { Ecard-Rocha } \\
(2013) \\
\text { Brazil }\end{array}$ & English & $\begin{array}{l}\text { Relationship } \\
\text { between motor } \\
\text { characteristics, } \\
\text { dermatoglyphics } \\
\text { for physical } \\
\text { qualities and } \\
\text { brain electro of } \\
\text { pre-and post- } \\
\text { menarche girls }\end{array}$ & \begin{tabular}{|l} 
Cross- \\
sectional, \\
descriptive
\end{tabular} & $\begin{array}{l}N=45 \text { girls } \\
10-14 \text { years } \\
21 \text { identified as } \\
\text { pre- menarche }\end{array}$ & MQs & Pediatry \\
\hline $\begin{array}{l}\text { Deprez } \\
(2014) \\
\text { Belgium }\end{array}$ & English & $\begin{array}{l}\text { Model of } \\
\text { development } \\
\text { including } \\
\text { maturity status, } \\
\text { body } \\
\text { composition and } \\
\text { motor } \\
\text { coordination }\end{array}$ & $\begin{array}{l}\text { Longitudinal } \\
\text { (5 years, } \\
2007-2012)\end{array}$ & $\begin{array}{l}N=162 \text { male } \\
\text { soccer players, age } \\
11-14 \text { years }\end{array}$ & $\begin{array}{l}\text { MC, } \\
\text { WB,JS,MS } \\
\text { raw scores }\end{array}$ & Sport sciences \\
\hline $\begin{array}{l}\text { Fransen } \\
(2014) \\
\text { Belgium }\end{array}$ & English & $\begin{array}{l}\text { Validity between } \\
\text { the BOTMP- } \\
\text { BOT and KTK }\end{array}$ & $\begin{array}{l}\text { Cross } \\
\text { sectional }\end{array}$ & $\begin{array}{l}N=2485(1300 \\
\text { boys }), \\
\text { 6-11 years }\end{array}$ & MQs & Sport sciences \\
\hline $\begin{array}{l}\text { Olesen } \\
(2014) \\
\text { Denmark }\end{array}$ & English & $\begin{array}{l}\text { Descriptions of } \\
\text { gender } \\
\text { differences in } \\
\text { motor skills, PA, } \\
\text { ethnicity, parents } \\
\text { education and } \\
\text { preschool }\end{array}$ & \begin{tabular}{|l|} 
Cross \\
sectional
\end{tabular} & $\begin{array}{l}N=607(299 \\
\text { boys }) \\
\text { mean age } 5.8 \\
\text { years }\end{array}$ & MQs & Sport sciences \\
\hline $\begin{array}{l}\text { Freitas } \\
(2015) \\
\text { Portugal }\end{array}$ & English & $\begin{array}{l}\text { Relationships } \\
\text { between age, } \\
\text { body size, } \\
\text { fundamental } \\
\text { motor skills and } \\
\text { motor } \\
\text { coordination }\end{array}$ & $\begin{array}{l}\text { Cross } \\
\text { sectional }\end{array}$ & $\begin{array}{l}N=429(213 \\
\text { boys }) \\
7-10 \text { years }\end{array}$ & $\begin{array}{l}\text { MC, raw } \\
\text { scores }\end{array}$ & Sport sciences \\
\hline
\end{tabular}

Notes: Small letters after the publication years in parentheses differentiate different publications in the reference list; The KTK refers to different editions of Kiphard, E.J., \& Schilling, F. (1974) Körperkoordinationstest für Kinder. Beltz test, Weinham; In cases where all four KTK test items (Walking Backwards [WB], Hopping for Height [HH], Jumping Sideways [JS], Moving Sideways $[M S])$ were not used, the items are mentioned; $M Q=$ Motor Quotient based on the KTK manual; $M C=$ refers to $M Q ; P A=$ physical activity; MOT 4-6 = Motoriktest für vier- bis sechsjährige Kinder; Movement-ABC = Movement Assessment Battery for Children; PDMS-2 = Peabody Developmental Motor Scales- Second Edition; TGMD-2 = Test of Gross Motor Development Second Edition; $M M T$ = Maastrichtse Motorik Test; BOTMP-BOT-2 = Bruininks-Oseretsky Test of Motor Proficiency.

Of the 46 articles, 27 were cross-sectional, nine were longitudinal designs with duration ranging from 7.5 weeks to 5 years, four were quasi-/experimental studies, two were reviews with no empirical component, one article reported a cluster-randomized-controlledintervention-trial, one utilized a prospective cohort, one was a retrospective design, and one was a reliability study. The articles were published between 1975 and 2015; 13 were in German. The 44 articles that reported empirical studies included between 17 (Jöhnk et al., 1999) and 7,175 (V. P. Lopes et al., 2012 b) participants with ages ranging from 5 to 26 years, and assigned to one to six groups. The total number of participants reported in the articles was 31,465 . The studies were conducted in 11 countries: Germany $(n=15)$, Belgium $(n=12)$, Portugal $(n=7$, representing different areas including the Azores and Madeira), the Netherlands $(n=3)$, Switzerland $(n=2)$, Austria $(n=1)$, Brazil $(n$ $=1)$, Costa Rica $(n=1)$, Denmark $(n=2)$, Finland $(n=1)$, and the United States $(n=1)$. 
Thirty-three articles reported the KTK MQs or percentile ranks based on the normative values given in the manual (Kiphard and Schilling, 1974, 2007). Of these articles, 20 provided categories of KTK performance, varying between MC disorder and very good MC. Six studies reported raw scores (Debrabant et al., 2012; Krombholz, 2011; V. P. Lopes et al., 2012b; Vandendriessche et al., 2012), and six articles reported only the scores for JS.

\section{Categorization}

The articles fell into six categories according to their main purposes and use of the KTK or some of the items in populations with typical development and with special needs (Table 2). Seventeen studies in populations with special needs used the KTK to investigate the associations between motor and other variables. Five of these studies investigated the associations between motor and other physiological variables, such as lead exposure (Winneke et al., 1982), the head size of children born prematurely and at term (Hebestreit et al., 2003), the neurodevelopment of preterm infants (Steiss et al., 2005), body function and composition of children with joint hypermobility (Hanewinkel-van Kleef et al., 2009), and the maturity statuses of national team soccer players (Vandendriessche et al., 2012). Twelve of the studies of populations with typical development focused on the associations between motor and other physiological variables, such as body composition (D'Hondt et al., 2011a; Freitas et al., 2015; V. P. Lopes, et al., 2012a; V.P. Lopes et al., 2012b; Martins et al., 2010; Olesen et al., 2015), physical activity and/or fitness (V. P. Lopes et al., 2011, 2012a; Vandendriessche et al., 2011), skeletal age (Freitas et al., 2015), injury risk (MartinDiener et al., 2013), and dermatoglyphic characteristics as indicators of genetic predispositions for physical qualities at maturation (Ecard Rocha et al., 2013). Associations between the KTK measured motor and physiological-social variables were investigated in three studies of typical populations. Interest was directed at body composition and leisure habits (Graf et al., 2004), sedentary and parental behavior (L. Lopes et al., 2012), and weight, sociodemographics, and behavioral factors (D'Hondt et al., 2011a). One study examined the associations between motor and psychological-physiological variables, such as perceptions of bodily competence and body mass index (Toftegaard-Stoeckel et al., 2010). Associations between motor and psychological-cognitive variables were reported in four articles. Two studies investigated psychiatric disorder markers in children with mental and behavioral disabilities (Baumann et al., 2004) and the IQ of children with chromosome deletion syndrome (Van Aken, Caeyenberghs et al., 2009). The two studies with typical populations examined performance in concentrated activities (Graf et al., 2003) and academic achievement (L. Lopes et al., 2013). Only one article reported on a study that examined associations between the KTK measured motor variables and factors pertaining to socioeconomic diversity (Prätorius and Milani, 2004).

The second biggest category of articles ( $n$ $=7$ ) was composed of studies that examined the effects of interventions or treatments on motor outcomes measured with the KTK. Among studies of populations with special needs, the studied effects on motor outcomes were creative movement and pantomime (Schneider, 1984), judo training (May et al., 2001), cochlear implant (Gheysen et al., 2008), and multidisciplinary obesity/overweight treatment (D'Hondt et al., 2011b). Types of interventions or treatments in the studies with typical populations were health education and physical activity (Graf and Dordel, 2011), grapho- and psychomotor treatment (Kranz et al., 2011), and different predictive response timing conditions (Debrabant et al., 2012).

Five articles reported on the use of the KTK or some of its items for diagnosis or identification purposes in populations with special needs (Deprez et al., 2014; Laucht et al., 2000; Lesigang and Aletsee, 1982; Schenck and Deegener, 1978; Stieh et al., 
1999). Three articles were reviews that analyzed motoscopic (von Neühauser, 1975), sensorimotor function (Jöhnk et al., 1999), or movement skill (Cools et al., 2009) assessment methods. One review included the implementation of a diagnostic follow-up study (Jöhnk et al., 1999). Three articles reported on measurement evaluation studies. The KTK's reliability in Spanish children (Camacho-Araya et al., 1990), the suitability of the KTK test norms for Dutch children (Smits-Engelsman et al., 1998), and convergent and discriminant validity between the KTK and the Bruininks-Oseretsky Test of Motor Proficiency 2 Short Form (BOT-2 Short Form; Bruininks and Bruininks, 2005; Fransen et al., 2014) were examined.

The smallest category was composed of two studies that focused on children's gross motor coordination per se. One study examined whether there was evidence of a decrease in motor performance (Krombholz, 2011), and the other study collected KTK reference values for a Flemish population (Vandorpe et al., 2011). 
Table 2. Categorization of articles according to their main purposes and the use of the Körperkoordinationstest für Kinder.

\begin{tabular}{|c|c|c|}
\hline & Typical populations & Special populations \\
\hline Category: & First author (year) & First author (year) \\
\hline \multicolumn{3}{|l|}{ Associations of Motor factors with } \\
\hline \multirow[t]{12}{*}{ Physiological variables } & Martins (2010) & Winneke (1982) \\
\hline & D'Hondt (2011a) & Hebestreit (2003) \\
\hline & V. P. Lopes (2011) & Steiss (2005) \\
\hline & Vandendriessche (2011) & Hanewinkel-van Kleef (2009) \\
\hline & V. P. Lopes (2012a) & Vandendriessche (2012) \\
\hline & V. P. Lopes (2012b) & \\
\hline & Laukkanen (2013) & \\
\hline & Martin-Diener (2013) & \\
\hline & V. P. Lopes (2013) & \\
\hline & Ecard-Rocha (2013) & \\
\hline & Olesen (2014) & \\
\hline & Freitas (2015) & \\
\hline \multirow[t]{3}{*}{ Physiological-social variables } & Graf (2004) & \\
\hline & L. Lopes (2012) & \\
\hline & D'Hondt (2013) & \\
\hline $\begin{array}{l}\text { Psychological-physiological } \\
\text { variables }\end{array}$ & Toftegaard-Stoeckel (2010) & \\
\hline \multirow{2}{*}{$\begin{array}{l}\text { Psychological-cognitive } \\
\text { variables }\end{array}$} & Graf (2003) & Baumann (2004) \\
\hline & L. Lopes (2013) & Van Aken (2009) \\
\hline Social variables & Prätorius (2004) & \\
\hline \multirow{4}{*}{$\begin{array}{l}\text { Intervention or treatment effectiveness on } \\
\text { motor outcomes }\end{array}$} & Graf (2011) & Schneider (1984) \\
\hline & Kranz (2011) & May (2001) \\
\hline & Debraband (2012) & Gheysen (2008) \\
\hline & & D'Hondt (2011b) \\
\hline \multirow[t]{5}{*}{ Diagnosis or identification } & & Schenck (1978) \\
\hline & & Lesigang (1982) \\
\hline & & Stieh (1999) \\
\hline & & Laucht (2000) \\
\hline & & Deprez (2014) \\
\hline \multirow[t]{2}{*}{ Review of methods } & Neühauser (1975) & \\
\hline & Cools (2008) & \\
\hline plus diagnosing & & Jöhnk (1999) \\
\hline \multirow[t]{2}{*}{ Measurement evaluation } & Camacho-Araya (1990) & Smits-Engelsman (1998) \\
\hline & Fransen (2014) & \\
\hline Motor evaluation & Krombholz (2011) & \\
\hline plus measurement evaluation & Vandorpe (2011) & \\
\hline
\end{tabular}




\section{Validity and reliability of the KTK}

Nine studies reported validity or reliability scores for the KTK in their target populations. Overall, the validity or reliability scores were reported to varying degrees of accuracy in these studies. Convergent and discriminant validity was reported in two studies. The KTK total score showed moderate correlations with the Movement ABC total score (Henderson and Sugden, 1992) ( $\mathrm{r}=$ 0.62-0.65) and the BOT-2 Short Form total score $(\mathrm{r}=0.61-0.64)$ in the different samples of 5- to 13-year-old Dutch and 6- to 11-yearold Belgian children, respectively (Fransen et al., 2014; Smits-Engelsman et al., 1998). When the test items, subsections, or composites were scrutinized separately, the lowest correlations were found between the KTK MS and Movement ABC balance $(r=$ 0.23, children without known motor difficulties) and the KTK MQ and BOT-2 Short Form fine motor composite score $(\mathrm{r}=$ 0.25, total sample of Belgian children). Correlations were strongest between the WB and Movement ABC Balance $(r=0.59$, children with known difficulties) and the MQ and BOT-2 Short Form gross motor composite score ( $\mathrm{r}=0.62$, group of 6-7-year-olds). Further, the percentages of children with typical development falling below the 15th percentile were $29 \%$ for the KTK versus $16 \%$ for the Movement $\mathrm{ABC}$, and the corresponding percentages for children with known difficulties were $68 \%$ for the KTK versus $59 \%$ for the Movement ABC (SmitsEngelsman et al., 1998). The percentages of children classified into score categories lower than the 5th and 15th percentiles and higher than the 85th and 95th percentiles were examined in a Belgian population. The results showed that the classification accuracy of the KTK was moderate for the 15 th percentile $(\kappa$ $=0.42$ ) and fair for the 5th, 85th, and 95th percentiles $(\kappa=0.31, \kappa=0.33, \kappa=0.33$, respectively). Fifty percent of children classified in $\leq 15$ th percentile by the KTK were likewise classified by the BOT-2 Short Form (Fransen et al., 2014).

Test-retest reliability correlations for the KTK in their target populations were reported in seven studies. Overall, the reliability coefficients $(r=0.60-0.99)$ showed moderate to high reliability (Camacho-Araya et al., 1990; Freitas et al., 2015; V. P. Lopes et al., 2011; 2012a, 2012b; Martins et al., 2010). Some studies reported reliability scores for the KTK total score and/or each test item separately. The highest re-test correlations were found for the total score $(r=0.89-0.94)$ and the lowest for the WB $(r=0.77-0.79)$ (Camacho-Araya et al., 1990; Olesen, et al., 2014). Internal consistency estimated with Cronbach's alpha was 0.95 (Camacho-Araya et al., 1990). Inter-rater reliability with a double Latin square design (two raters, two groups of subjects, two ways of giving directions and/or scoring, and re-testing after 8 days with the two raters switching the scoring method and the group of participants) was also high $(r=0.90-0.99)$. Analysis of variations indicated significant sources of variation $(\mathrm{F}>$ 52.13) by sex, grade, group of children, and treatment in samples $(\mathrm{n}=120$ and 90$)$ of Brazilian children (Camacho-Araya et al., 1990).

The following comments on the validity and/or reliability of the KTK were extracted from 13 papers and divided as follows: (1) The KTK can overestimate the number of children identified as having MC impairment (Hanewinkel van Kleef et al., 2009; SmitsEngelsman et al., 1998). (2) The KTK items represent movement product scores of balance and locomotor skills, and thus neither assesses MC all-inclusively nor is suitable for the assessment of MC development in children with clinical psychiatric disorders (Bauman et al., 2004; Cools et al., 2009; Jöhnk, 1999; Lesigang and Aletsee, 1982; V.P. Lopes et al., 2012a; Neuhaüser, 1975). (3) The norms were collected a long time ago and solely from German children. Thus, the cutoff scores were not heterogeneous enough to differentiate MC development at the low and high ends of the score distribution (V. P. Lopes et al., 2013; Prätorius and Milani, 2004; Schenk and Deegener, 1978; Vandorpe et al., 2011). 


\section{Discussion}

This review aimed to analyze studies that used the KTK test battery (Kiphard and Schilling, 1974, 2007). We identified 46 studies published in peer-reviewed articles over 40 years. The oldest paper (von Neühauser, 1975) reviewed motoscopic methods. Three articles were published in the 1980s, four in the 1990s, 12 in the 2000s, and 25 in the 2010s. The studies were mainly European. Since the KTK was created in Germany, the assessment's popularity in German-speaking countries was expected. A team of researchers reported on seven studies that had been conducted among 6- to 12-yearold Portuguese children (L. Lopes et al., 2012; 2013; V.P. Lopes et al., 2011, 2012a, 2012b, 2013; Martins et al., 2010). Two studies were from Latin America (Camacho-Araya, 1990; Ecard Rocha et al., 2013) and only one (published in German) from the United States (Schneider, 1984). To improve international comparisons, we recommend wider use of the KTK. Translation of the KTK manual into English might facilitate use in other cultures.

Overall, the sample sizes reported in the empirical studies varied according to the context and purpose. The number of participants, ranging from 17 to 347 , in the studies of populations with special needs was lower than in the studies with typical populations (range 45-7175). The KTK was most commonly used among children aged 612 years; 29 empirical studies investigated individuals between this age range. Since the KTK was standardized for 5- to 14-year-olds, its use in age groups outside this range, without ensuring assessment reliability in the target population, is questionable (Kranz et al., 2011; May et al., 2001). Eight different definitions, ranging from neutral ones, such as "scores" (e.g., Smits-Engelsman et al., 1998), to very specific ones, such as "dynamic balance" (e.g., D'Hondt et al., 2011a), were reported in the studies to describe the KTK outcomes. This indicates that the KTK was interpreted to measure somewhat different aspects. This finding strengthens the view that various terms exist in the literature to describe general and specific aspects in the motor domain and that uncertainty exists regarding an optimal definition (Logan et al., 2014). However, the fact that the KTK was considered a suitable battery for various purposes across such different fields as physical education, sport, health sciences, medicine, and biomechanics can be interpreted as strength of the KTK.

In the majority of the studies, the KTK results were reported as age- and genderstandardized MQs calculated from the four items and variously classified as indicators of the motor status of the individuals tested. Producing MQ outcomes was seen as a system of scoring that describes individuals' global $\mathrm{MC}$ in meaningful categories, and allows comparison with results from other studies (V. P. Lopes et al., 2012b; Toftegaard-Stoeckel et al., 2010). Since Kiphard and his colleagues developed the KTK in 5- to 14-year-old children, (Kiphard and Schilling, 2007, p. 7), it was logical that all 27 studies with typical populations were implemented in school or kindergarten settings. Perhaps, the KTK is suitable for schools, because it is reasonably quick and easy to administer. The participant groups in the 17 studies of populations with special needs differed in terms of special features. The KTK, since it is not sportspecific, was regarded as a suitable instrument for measuring the general motor level of different individuals and in special circumstances (Vandendriessche et al., 2012; Vandorpe et al., 2011).

The studies fell into six categories according to their main purposes and use of the KTK. Categorizing the studies was challenging due to the varying variables, such as background factors, field test, or interview outcomes and laboratory test samples, used in them. The biggest category was composed of studies that reported associations of MC with other factors. Of these associative studies, most investigated physiological factors. The studies frequently focused on children's body composition and physical activity or fitness, which could be expected in view of the global concern over the trend toward unhealthy weight and increased physical inactivity 
among children (Leech, et al., 2014). Surprisingly, only two articles in the category of intervention or treatment effectiveness studies reported on the implementation of programs promoting a healthy eating or increased physical activity (D'Hondt et al., 2011b; Graf and Dordel, 2011). Overall, the KTK was used in different and multifaceted designs to provide motor domain outcomes among other variables. As expected, this was the case in the studies of populations with special needs, where the designs differed widely. Since the KTK was developed for screening purposes, it was reasonable that we identified a category of studies that mainly diagnosed or identified different medical factors, including motor variables measured by the KTK. Scrutiny of the historical continuum of the studies that used the KTK revealed that the use of the KTK has now spread from special fields of medicine to education and the sport sciences.

On the whole, the KTK was deemed a "thoroughly" standardized and validated tool, and was given as an appendix in the articles along with a reference to the KTK manual. An undesired finding was that only nine studies examined the KTK's validity or reliability in the target populations. Overall, the re-test coefficients indicated good reliability for the test battery. Notwithstanding, we suggest that feasibility studies that ensure satisfactory validity and reliability be carried out in target populations. This is important in populations with special needs, in whom symptoms, morbidity status, and other individual features vary widely, and populations at an early phase of motor development, when intra- and interindividual differences are large and development is rapid (Gallahue et al., 2012). In fact, many authors criticized the validity or reliability of the KTK (Baumann et al., 2004; Jöhnk et al., 1999; Lesigang and Aletsee, 1982), but for some reason did not report addressing these concerns in their target groups. For example, the KTK was not seen as an inaccurate method of diagnosing minimal cerebral palsy (Lesigang and Aletsee, 1982) or estimating the number of children suffering from brain impairments (Smits-Engelsman et al., 1998). Consequently, the use of additional tests was advised, including by authors who questioned the validity of the KTK because it produces movement product scores and is limited to balance and locomotor aspects among the fundamental motor skills (Cools et al., 2009; V.P. Lopes et al., 2012a; Neuhäuser, 1975). We agree with this view. Recently, researchers have started to combine different test batteries for more specific measures of MC and fundamental motor skills to compensate for the limitations imposed by a single test battery (Freitas et al., 2015). We support this trend.

The few studies that tested the validity of the KTK all came to the conclusion, regardless of the specific population studied, that the number of children identified as having motor difficulties was considerably higher for the KTK than for the other test batteries designed for the same purpose (Fransen et al., 2014; Hanewinkel van Kleef et al., 2011; SmitsEngelsman et al., 1998). Children's living conditions and lifestyles have changed from the 1970s to today. Therefore, classifying children's motor characteristics based on normative values collected four decades ago may give a different picture of the present-day reality, as was discussed in the literature (Baumann et al., 2004; Smits-Engelsman et al., 1998; Vandorpe et al., 2011). As confirmation, the KTK showed moderate validity when the test discriminated between children with relatively poor motor proficiency and those with average to good proficiency but only fair validity when the KTK was used to classify children with high or very poor proficiency. This may indicate that the KTK categorizes children incorrectly (Fransen et al., 2014). Consequently, careful interpretation of norm-referenced performances is advised, since normative data are often based on limited samples (Cools et al., 2009). This notion applies to the KTK, as its normative data were collected from children who shared the same culture across a limited geographic area. Possible methods for remedying this limitation include collecting international reference values or examining cultural sensitivity before using the test battery 
in a particular region (Tripathi et al., 2008). In sum, the results of this review study lead us to recommend to carefully pre-examine the potential test batteries and/or test items to be used or combined before choosing a particular measure for a particular purpose. Since the original aim of the KTK was to identify and, where necessary, recommend further evaluations of children with brain damage, as well as assist in designing appropriate means of rehabilitation for this specific group, researchers and others who use the battery for purposes unrelated to this aim need to include the evaluation of validity for their target groups.

\section{Perspective}

The KTK was originally developed to identify children with brain damage. It is now used as a measure for MC for various research purposes in children with different development in multiple contexts. From the users' perspective, the KTK was regarded to be (1) easy to administer, (2) usable in children with and without impairments, (3) already in wide use in Europe and becoming increasingly popular, (4) carefully standardized in German population, (5) a nonsport/skill-specific measure, and (7) based on a meaningful scoring system that enables cross-study comparisons. The KTK was criticized because it is limited to balance and locomotor aspects, has outdated norms with cutoff scores that are not heterogeneous enough to differentiate MC development. The KTK may overestimate the number of children identified as having MC problems. Based on the review conducted, we recommend the use of KTK raw scores when the interest is to follow MC development and when reporting research. We also warrant examination of the validity of the KTK for different purposes and in different target populations.

\section{Supplementary files}

Supplementary file 1. Description of the test items (1-4) and scoring of the The
Körperkoordinationstest für Kinder (KTK) (Kiphard and Schilling, 1974, 2007).

\section{References}

Baumann, C., Löffler, C., Curic, A., Schmid, E., \& von Aster, M. (2004). Motorische Entwicklung und kinderpsychiatrische Störungen [Motor skills and psychiatric disturbances in children]. Psychiatrische Praxis, 31(8), 395-399.

Bruininks, R., \& Bruininks, R. (2005). Bruininks-Oseretsky Test of Motor Proficiency, second edition (BOT-2). Minneapolis, MN: Pearson Assessment.

Camacho-Araya, T., Woodburn, S. S., \& Boschini, C. (1990). Reliability of the prueba de coordinación corporal para niños [Body coordination test for children]. Perceptual and Motor Skills, 70, 832-834

Cools, W., De Martelaer, K., Samaey, C., \& Andries, C. (2009). Movement skill assessment of typically developing preschool children: A review of seven movement skill assessment tools. Journal of Sports Science \& Medicine, 8(2), 154-168.

Debraband, J., Gheysen, F., Vingerhoets, G., \& van Waelvelde, H. (2012). Agerelated differences in predictive response timing in children: Evidence from regularly relative to irregularly paced reaction time performance. Human Movement Science, 31(4), 801-810. doi:10.1016/j.humov.2011.09.006.

D'Hondt, E., Deforche, B., Gentier, I., De Bourdeaudhuij, I., Vaeyens, R., Philippaerts, R., \& Lenoir, M. (2013). A longitudinal analysis of gross motor coordination in overweight and obese children versus normal-weight peers. International Journal of Obesity, 37(1), 61-67. doi:10.1038/ijo.2012.55.

D'Hondt, E., Deforche, B., Vaeyens, R., Vandorpe, B., Vandendriessche, J., Pion, J., . . Lenoir, M. (2011a). Gross motor coordination in relation to weight status and age in 5- to 12-year-old boys and girls: A cross-sectional study. 
International Journal of Pediatric Obesity, 6(2-2), e556-e564. doi:10.3109/17477166.2010.500388.

D'Hondt, E., Gentier, I., Deforche, B., Tanghe, A., Bourdeaudhuij, I. D., \& Lenoir, M. (2011b). Weight loss and improved gross motor coordination in children as a result of multidisciplinary residential obesity treatment. Obesity, 19(10), 1999-2005. doi:10.1038/oby.2011.150.

Deprez, D., Valente-dos-Santos, J., Coelho e Silva, M., Lenoir, M., Philippaerts, R.M., \& Vaeyens, R. (2014). Modeling developmental changes in the Yo-Yo Intermittent Recovery Test Level 1 in Elite Pubertal Soccer Players. International Journal of Sports Physiology and Exercise, 9, 1006-1012. doi: 10.1123/ijspp.2013-0368.

Ecard Rocha, L., Roquetti Fernandes, P., \& Fernandes Filho, J. (2013). Motor features, dermatoglyphic and egg periods of girls in pre and post menarche. Sport Logia, 9(1), 34-45.

Fransen, J., D'Hondt, E., Bourgois, J., Vaeyens, R., Philippaerts, R., \& Lenoir, M. (2014). Motor competence assessment in children: Convergent and discriminant validity between the BOT-2 Short Form and KTK testing batteries. Research in Developmental Disabilities 35, 1375-1383. doi: 10.1016/j.ridd.2014.03.011.

Freitas, D.L., Lausen, B., Maia, J.A., Lefevre, J., Gouvela, E.R., Thomis, M....Malina, R.M. (2015) Skeletal maturation, fundamental motor skills and motor coordination in children 7-10 years. Journal of Sport Sciences, 33(9),doi: 10.1080/02640414.2014.977935.

Gallahue, D. L., Ozmun, J. C., \& Goodway, J. (2012). Understanding motor development: Infants, children, adolescents, adults (7th ed.). New York: McGraw-Hill, p. 260.

Gheysen, F., Loots, G., \& van Waelvelde, H. (2008). Motor development of deaf children with and without cochlear implants. Journal of Deaf Studies and
Deaf Education, 13(2), 215-224. doi:10.1093/deafed/enm053.

Graf, C., \& Dordel, S. (2011). Das CHILT-IProjekt (Children's Health InterventionaL Trial) Eine multimodale Maßnahme zur Prävention von Bewegungsmangel und Übergewicht an Grundschulen [The CHILT-I project (children's health interventional trial). A multicomponent intervention to prevent physical inactivity and overweight in primary schools].

Bundesgesundheitsblatt,

Gesundheitsforschung,

Gesundheitsschutz, 54(3), 313-321. doi:10.1007/s00103-010-1230-9.

Graf, C., Koch, B., Klippel, S., Büttner, S., Coburger, S., Christ, H.,...Dordel, S. (2003). Zusammenhänge zwischen körperliche Aktivität und Konzentration in Kindesalter- Eingangsergebnisse des CHILTS-Projecktes [Correlation between physical activities and concentration in children results of the CHILT project]. Deutsche Zeitschrift für Sportmedizin, 54, 242-246.

Graf, C., Koch, B., Kretschmann-Kandel, E., Falkowski, G., Christ, H., Coburger, S. . . . Dordel, S. (2004). Correlation between BMI, leisure habits and motor abilities in childhood (CHILT-project). International Journal of Obesity and Related Metabolic Disorders, 28(1), 2226.

Hanewinkel-van Kleef, Y. B., Helders, P. J. M., Takken, T., \& Engelbert, R. H. (2009). Motor performance in children with generalized hypermobility: The influence of muscle strength and exercise capacity. Pediatric Physical Therapy, 21(2), 194-200. doi:10.1097/PEP.0b013e3181a3ac5f.

Hebestreit, H., Schrank, W., Schrod, L., Strassburg, H., \& Kriemler, S. (2003). Head size and motor performance in children born prematurely. Medicine and Science in Sports and Exercise, 35(6), 914-922.

Henderson, S.E., \& Sugden, D.A., 1992. Movement Assessment Battery for 
Children. London: Psychological Corporation.

Jöhnk, K., Kuhtz-Buschbeck, J. P., Stolze, H., Serocki, G., Kalwa, S., Ritz, A., . . . Illert, M. (1999). Assessment of sensorimotor functions after traumatic brain injury (TBI) in childhood methodological aspects. Restorative Neurology and Neuroscience, 14(2), 143-152.

Kranz, I., Sammann, K., Amft, S., \& Vetter, M. (2011). Effekte einer grafomotorisch ausgerichteten psychomotorischen Intervention bei Kindern im Vorschulalter. Psychologie in Erziehung Und Unterricht, 58(2), 139-151.

Kiphard, E.J. \& Schilling, F. (1974). Körperkoordinationstest für Kinder. Belz test, Weinham.

Kiphard, E.J. \& Schilling, F. (2007). Körperkoordinationstest für Kinder 2, überarbeitete und ergänzte Aufgabe. Beltz test, Weinham.

Krombholz, H. (2011). Haben sich motorische Leistungen von 3- bis 7-jährige Jungen und Mädchen im Zeitraum von 1973 bis 2001 verschlechtert? [Is there evidence of a decrease in motor performance of 3to 7-year-old boys and girls in 2001 compared to those of the 1970s?] Zeitschrift für Sportpsychologie, 18(4), 161-171.

Laucht, M., Esser, G., \& Schmidt, M. H. (2000). Entwicklung von Risikokindern im Schulalter: Die langfristigen Folgen frühkindlicher Belastungen [Developmental outcome of at-risk children at school age: Long-term sequelae of early risk factors]. Zeitschrift für Entwicklungspsychologie und Pädagogische Psychologie 32(2), 5969.

Laukkanen, A., Pesola, A., Havu, M., Sääkslahti, A. \& Finni, T. (2014). Relationship between habitual physical activity and gross motor skills is multifaceted in 5- to 8-year-old children. Scandinavian Journal of Medicine \& Science in Sports, 24, e102-e110, doi: $10.1111 / \mathrm{sms} .12116$.
Leech, R. M., McNaughton, S. A., \& Timperio, A. (2014). The clustering of diet, physical activity and sedentary behavior in children and adolescents: A review. International Journal of Behavioral Nutrition and Physical Activity, 11(4), doi:10.1186/1479-586811-4.

Lesigang, C., \& Aletsee, S. (1982). Motorskopisch-neurologische und motormetrische Paralleluntersuchung von $192 \quad$ Sprachheilschülern [Motoscopic-neurological and motometric investigation in children with speech disturbances]. Pädiatrie Und Pädologie, 17(2), 353-360.

Logan, S. W., Robinson, L. E., Rudisill, M. E., Wadsworth, D. D., \& Morera, M. (2014). The comparison of school-age children's performance on two motor assessments: The Test of Gross Motor Development and The Movement Assessment Battery for children. Physical Education and Sport Pedagogy, $\quad$ 19(1), 48-59. doi:10.1080/17408989.2012.726979.

L. Lopes, Santos, R., Pereira, B., \& Lopes, V. (2013). Associations between gross motor coordination and academic achievement in elementary school children. Human Movement Science, 32(1), 9-20.

L. Lopes, Santos, R., Pereira, B., \& Lopes, V.P. (2012). Associations between sedentary behaviour and motor coordination in children. American Journal of Human Biology, 24(6), 746752. doi: 10.1002/ajhb.22310.

V. P. Lopes, Rodrigues, L. P., Maia, J. A. R., \& Malina, R. M. (2011). Motor coordination as predictor of physical activity in childhood. Scandinavian Journal of Medicine \& Science in Sports, 21(5), 663-669.

V. P. Lopes, Maia, J. A. R., Rodrigues, L. P., \& Malina, R. (2012a). Motor coordination, physical activity and fitness as predictors of longitudinal change in adiposity during childhood. European Journal of Sport Science, 
12(4),

384-391. doi:10.1080/17461391.2011.566368.

V. P. Lopes, Stodden, D. F., Bianchi, M. M., Maia, J. A. R., \& Rodrigues, L. P. (2012b). Correlation between BMI and motor coordination in children. Journal of Science and Medicine in Sport, 15(1), 38-43. doi:10.1016/j.jsams.2011.07.005.

V.P. Lopes, Stodden, D.F., \& Rogriques, L.P. (2013). Weight status is associated with cross-sectional trajectories of motor coordination across childhood. Child: Care, Health and Development, 40(6), 891-899. doi: 10.1111/cch.12127.

Martin-Diener, E., Wanner, M., Kriemler, S., \& Martin, B.W. (2013). Associations of objectively assessed levels of physical activity, aerobic fitness and motor coordination with injury risk in school children aged 7-9 years: cross-sectional study. BMJ open 2013, 3: e003086, 1-7. doi: 10.1136/bmjopen-2013-003086.

Martins, D., Maia, J., Seabra, A., Garganta, R., Lopes, V., Katzmarzyk, P., \& Beunen, G. (2010). Correlates of changes in BMI of children from the Azores Islands. International Journal of Obesity, 34(10), 1487-1493. doi:10.1038/ijo.2010.56.

May T.W., Baumann C., Worms L., Koring W., \& Aring R. (2001). Effects of judo training on physical coordination and body sway in adolescents and young adults with multiple impairments and epilepsy. Deutsche Zeitschrift für Sportmedizin 52, 245-251.

von Neühauser, G. (1975). Zur Bedeutung motorisher Tests für die Entwicklungsneurologische Diaknostik. Forschricht Medicine, 93, 1159-1166.

Olesen, L.G., Kristensen, P.L., Ried-Larsen, M., Grøntved, A., \& Froberg, K. (2015). Physical activity and motor skills in children attending 43 preschools: a cross-sectional study. BMC Pediatrics, 14, 229. doi:10.1186/1471-2431-14-229.

Prätorius, B., \& Milani, T.L. (2004). Motorische Leistungsfähigkeit bei Kindern: Koordinations- und Gleichgewichtsfähigkeit: Untersuchung des Leistungsgefälleszwischen Kindern mit verschiedenen

Sozialisationsbedingungen [Motor abilities of children: Abilities of coordination and balance: examination of differences between children of different social groups]. Deutsche Zeitschrift für Sportmedizin, 55(7), 172175.

Schenck, K., \& Deegener, G. (1978). In the diagnostic efficiency of the bodycoordination-test for children (KTK) (author's transl). Monatsschrift für Kinderheilkunde, 126(1), 40-43.

Schneider, F. J. (1984). Effects of classes in "creative movement and pantomime" and "badminton" on total-body coordination in older dyslexic boys. Die Rehabilitation, 23(4), 148-154.

Smits-Engelsman, B., Henderson, S., \& Michels, C. (1998). The assessment of children with developmental coordination disorders in the Netherlands: The relationship between the Movement Assessment Battery for Children and the Körperkoordinationstest für Kinder. Human Movement Science, 17(4-5), 699-709. doi:10.1016/S01679457(98)00019-0.

Steiss, J., Langner, C., \& Neuhauser, G. (2005). Neurologische Entwicklung, Körperkoordination und Visuomotorik bei ehemals gesund entlassenen frühgeborenen Kindern im Alter von neun bis zwölf Jahren [Development of preterm infants at the age of 9-12 years after normal neonatal neurological examination]. Kindheit Und Entwicklung, 14(3), 163-168. doi:10.1026/0942-5403.14.3.163.

Stieh, J., Kramer, H. H., Harding, P., \& Fischer, G. (1999). Gross and fine motor development is impaired in children with cyanotic congenital heart disease. Neuropediatrics, 30(2), 77-82.

Toftegaard-Stoeckel, J., Groenfeldt, V., \& Andersen, L. B. (2010). Children's selfperceived bodily competencies and associations with motor skills, body mass index, teachers' evaluations, and 
parents' concerns. Journal of Sports Sciences, 28(12), 1369-1375. doi:10.1080/02640414.2010.510845.

Tripathi, R., Joshua, A.M., Kotian, M.S., \& Tedla, J.S. (2008). Normal motor development of Indian children on Peabody Developmental Motor Scales-2 (PDMS-2). Pediatric Physical Therapy, 20(2), 167-172.

Van Aken, K., Caeyenberghs, K., SmitsEngelsman, B., \& Swillen, A. (2009). The motor profile of primary school-age children with a 22q11.2 deletion syndrome (22q11.2DS) and an age- and IQ-matched control group. Child Neuropsychology: A Journal on Normal and Abnormal Development in Childhood and Adolescence, 15(6), 532542. doi:10.1080/09297040902740678.

Vandendriessche, J. B., Vaeyens, R., Vandorpe, B., Lenoir, M., Lefevre, J., \& Philippaerts, R. M. (2012). Biological maturation, morphology, fitness, and motor coordination as part of a selection strategy in the search for international youth soccer players (age 15-16 years). Journal of Sports Sciences, 30(15), 1695-1703. doi:10.1080/02640414.2011.652654.

Vandendriessche, J. B., Vandorpe, B., Coelhoe-Silva, M. J., Vaeyens, R., Lenoir, M., Lefevre, J., \& Philippaerts, R. M. (2011). Multivariate association among morphology, fitness, and motor coordination characteristics in boys age 7 to 11. Pediatric Exercise Science, 23(4), 504-520.

Vandorpe, B., Vandendriessche, J., Lefevre, J., Pion, J., Vaeyens, R., Matthys, S., . . Lenoir, M. (2011). The Körperkoordinationstest für kinder: Reference values and suitability for 612-year-old children in Flanders. Scandinavian Journal of Medicine \& Science in Sports, 21(3), 378-388. doi:10.1111/j.1600-0838.2009.01067.x.

Winneke, G., Hrdina, K. G., \& Brockhaus, A. (1982). Neuropsychological studies in children with elevated tooth-lead concentrations. I. pilot study.
International Archives of Occupational and Environmental Health, 51(2), 169183. 